\title{
FUNCTIONAL CHARACTERIZATIONS OF THE FIELD OF VALUES AND THE CONVEX HULL OF THE SPECTRUM
}

\author{
CHARLES R. JOHNSON
}

\begin{abstract}
The only compact, convex set-valued homogeneous and translatable function of square complex matrices which is an indicator function for the matrices with positive semidefinite real part is the classical field of values. An analogous characterization of the convex hull of the spectrum is given.
\end{abstract}

Considered as a function from square complex matrices into subsets of the complex plane, many functional properties of the usual field of values

$$
F(A) \equiv\left\{x^{*} A x: x^{*} x=1\right\}, \quad A \in M_{n}(C),
$$

are known. For example, if $A$ and $B$ are any square complex matrices of the same size, then:

(1) $F(A)$ is a compact set;

(2) $F(A)$ is a convex set;

(3) $F(A+\alpha I)=F(A)+\alpha$ for any complex number $\alpha$;

(4) $F(\alpha A)=\alpha F(A)$ for any nonzero complex number $\alpha$;

(5) $F(A)$ is contained in the closed right half-plane if and only if $A+A^{*}$ is positive semidefinite;

(6) the set of eigenvalues $\sigma(A) \subseteq F(A)$;

(7) $F\left(U^{*} A U\right)=F(A)$ if $U$ is unitary;

(8) $F(A+B) \subseteq F(A)+F(B)$;

(9) $F(A \oplus B)$ is the convex hull of $F(A) \cup F(B)$;

(10) $F\left(A_{0}\right) \subseteq F(A)$ if $A_{0}$ is a principal submatrix of $A$; and

(11) $F(A)$ is the convex hull of $\sigma(A)$ if $A$ is normal.

There has been interest recently in generalizations of the field of values achieved by postulating as axioms on a complex-set-valued function of square matrices some subset of the known properties of $F$ [3], [4]. In the past, generalizations have also been achieved via norms other than the Euclidean norm. The question we address here is what, if any, subsets of the known properties of $F$ uniquely define $F$. It is clear that in this regard some of the above functional properties of $F$ are redundant. For example, we have

REMARK 1. Property (6) above follows from properties (1), (3), (4), (7), and

Received by the editors March 9, 1976.

AMS (MOS) subject classifications (1970). Primary 15-00, 15A15, 15A63, 02H 10.

Key words and phrases. Field of values, convex hull of spectrum.

(c) American Mathematical Society 1977 
(10). Suppose $\beta \in \sigma(A)$. Then for some unitary $U$, the matrix $U^{*} A U$ is upper triangular with $\beta$ in the 1,1 position. Then, by (7) and (10), it is enough to show that $\beta \in F([\beta])$, and, because of (3), it suffices to show that $0 \in$ $F([0])$. However, because of (4), $F([0])=\alpha F([0])$ for any $\alpha \neq 0$ and there are only 2 sets possessing this property: $\{0\}$ and the entire plane. The latter is precluded by (1).

REMARK 2. Also (7) follows from (1)-(5) by using, for example, the fact that the class of matrices with positive semidefinite real part

$$
\left(\operatorname{Re}(A)=\frac{1}{2}\left(A+A^{*}\right)\right)
$$

is conjunctively invariant.

Our main result is that there is a subset of five of (1)-(11) which characterizes $F$. Furthermore, we show by a sequence of examples that no one of these five may be deleted while retaining the property of characterizing $F$. It would be of interest to know what other sets of properties characterize $F$ and to know, for example, more about the classes of functions which satisfy various subsets of (1)-(11).

THEOREM 1. Suppose $S$ is a function which maps square complex matrices (of all orders) into the subsets of the complex plane. Then $S=F$, the classical field of values, if and only if for all $A$

(i) $S(A)$ is a compact set;

(ii) $S(A)$ is a convex set;

(iii) $S(A+\alpha I)=S(A)+\alpha$ for any complex number $\alpha$;

(iv) $S(\alpha A)=\alpha S(A)$ for any nonzero complex number $\alpha$; and

(v) $S(A)$ is contained in the closed right half-plane if and only if $A+A^{*}$ is positive semidefinite.

Proof. If $S=F$, then (i)-(v) are well known to hold.

To prove the converse, we first show that $F(A) \subseteq S(A)$ for an arbitrary $A$. Suppose, to the contrary, that $\beta \in F(A)$ and $\beta \notin S(A)$ for some $A$. Then, because of (i) and (ii), there exist $\alpha_{1} \neq 0$ and $\alpha_{2}$ such that $\operatorname{Re}\left(\alpha_{1} \beta+\alpha_{2}\right)<0$ while $\alpha_{1} S(A)+\alpha_{2}$ is contained in the closed right half-plane. However, $\alpha_{1} S(A)+\alpha_{2}=S\left(\alpha_{1} A+\alpha_{2} I\right)$ because of (iii) and (iv) so that we have $\operatorname{Re}\left(\beta^{\prime}\right)<0$ while $S\left(A^{\prime}\right)$ lies in the closed right half-plane, where $\alpha_{1} \beta+\alpha_{2}=$ $\beta^{\prime} \in F\left(A^{\prime}\right)$ and $A^{\prime}=\alpha_{1} A+\alpha_{2} I$. Then, by (v) $A^{\prime}+A^{\prime *}$ is positive semidefinite. This, however, contradicts the fact that $F\left(A^{\prime}\right)$ is not contained in the closed right half-plane so that we may conclude that $F(A) \subseteq S(A)$.

Since the containment $F(A) \subseteq S(A)$ depends only on the fact that $S(A)$, as well as $F(A)$, satisfies (i)-(v), the above line of proof also means that $S(A) \subseteq F(A)$ for an arbitrary $A$. We conclude that $S=F$ under the assumption of (i)-(v) on $S$. This completes the proof.

EXAmple 1. No subset of four of the five conditions of Theorem 1 is sufficient to characterize the field of values $F$. 
(a) Conditions (i)-(iv) are satisfied by letting $S(A)$ be the convex hull of the eigenvalues of $A$.

(b) Conditions (i), (ii), (iii) and (v) are satisfied by letting $S(A)$ be $\{z$ : $z \in F(A)$ and $\left.\operatorname{Re}(z)=\min _{w \in F(A)} \operatorname{Re}(w)\right\}$.

(c) Conditions (i), (ii), (iv) and (v) are satisfied by letting $S(A)$ be the convex hull of $F(A) \cup\{\operatorname{Tr}(A)\}$.

(d) Conditions (i), (iii), (iv) and (v) are satisfied by letting $S(A)$ be the boundary of $F(A)$.

(e) Conditions (ii)-(v) are satisfied by letting $S(A)$ be the interior of $F(A)$ whenever the interior of $F(A)$ is nonempty and letting $S(A)$ be $F(A)$ otherwise.

None of these definitions of $S$ coincides with $F$ for all $A$.

REMARK 3. Since (i)-(v) characterize $F$, all other functional properties of $F$ follow from them.

A square complex matrix is called (positive) stable if each of its eigenvalues has positive real part and (positive) semistable if each of its eigenvalues has nonnegative real part. A slight alteration of (v) above yields a parallel characterization of the convex hull of the spectrum.

THEOREM 2. Suppose $S$ is a function which maps square complex matrices (of all orders) into the subsets of the complex plane. Then $S=C$, the convex hull of the eigenvalues, if and only if for all $A$

(i) $S(A)$ is a compact set;

(ii) $S(A)$ is a convex set;

(iii) $S(A+\alpha I)=S(A)+\alpha$ for any complex number $\alpha$;

(iv) $S(\alpha A)=\alpha S(A)$ for any nonzero complex number $\alpha$; and

$\left(\mathrm{v}^{\prime}\right) S(A)$ is contained in the closed right half-plane if and only if $A$ is (positive) semistable.

\section{Proof. Similar to that of Theorem 1.}

REMARK 4. In view of Theorems 1 and 2, the convex hull of the eigenvalues is to the theory of stable matrices what the field of values is to the theory of matrices with positive definite real part. See [2] for another point of view on the relation between the field of values and the convex hull of the spectrum.

Actually, if (v) and $\left(v^{\prime}\right)$ are strengthened slightly, axiom (i) may be deleted from Theorems 1 and 2. An essentially equivalent line of proof yields

THEOREM 3. Suppose $S$ is a function which maps square complex matrices into the subsets of the complex plane. Then $S=F$, the classical field of values, if and only if for all $A$

(ii) $S(A)$ is a convex set;

(iii) $S(A+\alpha I)=S(A)+\alpha$ for any complex number $\alpha$;

(iv) $S(\alpha A)=\alpha S(A)$ for any nonzero complex number $\alpha$; and 
(vi) $S(A)$ is contained in the open right half-plane if and only if $A+A^{*}$ is positive definite,

and

THEOREM 4. Suppose $S$ is a function which maps square complex matrices into the subsets of the complex plane. Then $S=C$, the convex hull of the eigenvalues, if and only if for all $A$

(ii) $S(A)$ is a convex set;

(iii) $S(A+\alpha I)=S(A)+\alpha$ for any complex number $\alpha$;

(iv) $S(\alpha A)=\alpha S(A)$ for any nonzero complex number $\alpha$; and

(vi') $S(A)$ is contained in the open right half-plane if and only if $A$ is (positive) stable.

Clearly assumptions (vi) and (vi') are quite strong as many different functions $S$ (e.g. the Geršgorin type set of [1]) satisfy (ii), (iii) and (iv) as well as several other properties of $F$.

REMARK 5. The class of functions satisfying any one (and thus any subset) of the properties indicated by (1)-(11) is closed under the two operations:

(a) set intersection: $S(A) \equiv S_{1}(A) \cap S_{2}(A)$ [except for (8)]; and

(b) $S(A) \equiv \operatorname{Co}\left\{S_{1}(A) \cup S_{2}(A)\right\}$.

Thus, a lattice structure and minimal elements might be considered.

\section{REFERENCES}

1. C. R. Johnson, A Geršgorin inclusion set for the field of values of a finite matrix, Proc. Amer. Math. Soc. 41 (1973), 57-60. MR 47 \#6738.

2. _ A Lyapunov theorem for angular cones, J. Res. Nat. Bur. Standards Sect. B 78 (1974), 7-10. MR 48 \#11161.

3. B. Saunders and H. Schneider, A symmetric numerical range for matrices (to appear).

4. C. Zenger, Minimum subadditive inclusion domains for the eigenvalues of matrices (to appear).

Institute for Physical Science and Technology, University of Maryland, College PARK, MARYLAND 20742

Applied Mathematics Division, National Bureau of Standards, Washington, D.C. 20234 